\title{
Inter Cell Scheduling Based on Queuing Networks
}

\author{
Jie LV,Juan-Juan ZHANG, Wen-Min HAN
}

\author{
School of Economics and Management, Jiangsu University of Science \& Technology, Zhenjiang, \\ Jiangsu, China \\ Lvjie0511@126.com
}

Keywords: Inter cell, Queuing network, Virtual cellular manufacturing, Batch size.

\begin{abstract}
In virtual cellular manufacturing, the processing lines are more complex, products are multi-varieties and small quantities, jobs spend $90 \%$ of the time waiting for the machine to be processed is common. In order to reduce the waiting time, this paper study the inter cell scheduling based on queuing network. The virtual cellular manufacturing system is seen as a queuing network, and the input parameters of each node within the network are calculated by the external arrival rate. Under the strategy of batch service. Scheduling target is minimize the total processing time, maximize the minimum of equipment load. Batch service queuing network model is established. The model is solved by the immune optimization algorithm, which achieves the shortest processing time, and avoids the parts waiting. Finally, taking the actual production process of ship enterprises as an example, it is proved that the batch service queuing network method is feasible and efficient.
\end{abstract}

\section{Introduction}

Modern manufacturing environment is characterized by intense of market competition, diversity of products and short life period, rapid changes in customer demand, which lead to efficient utilization of resource and competitive reaction of trying to seize the market[1].Cell manufacturing system (CMS) has the advantages of shortening setup time, production time, reducing WIP inventory, reducing cost of material handling, improving quality of product and controlling production, which leads to improving efficiency and flexibility[2]

Cell scheduling can be divided into cell internal scheduling and scheduling between multiple cells [3], the problem of internal scheduling has been studied by quite a few scholars. Mak et al. [4] studied the dynamic scheduling problem of parts in Virtual Manufacturing Cell under multi - period of determining demand. For the problem of inter-cell scheduling, Tang et al. [5]studied the joint decision of cell formation and scheduling, and considered the movement between the cells, which was solved by the Lagrange relaxation algorithm. Moghaddam[6]constructed an integer programming mathematical model, and proposed a sub-heuristic algorithm based on decentralized search to solve the model.

Therefore, some scholars use the queuing network method to solve the problem of cell scheduling. Hasenbein[7]and Harrison[8] treat each batch part as a work piece, and use an ordinary single-server queuing network to find the approximate solution. The queuing network method shortens the waiting time of parts. J.G.Dait[9]proved that the strategy of batch scheduling can be solved by a single-service queuing network with a certain key strategy in cell manufacturing. Zhu Dengjie et al.[10] proposed an improved open-loop queuing network model for the characteristics of automated material transportation systems of wafers manufacturing. The queuing network model mentioned above is based on batch which is fixed, and assumes that the service rate is constant. Liu $\mathrm{Li}$ [11] used the queuing theory to study the dynamic scheduling problem of cloud task. Considering the uncertain parameters of service rate of the task arrival and network transmission, which lead to the improvement of average response time of the whole cell scheduling. Laxmi, Seleshi[12]consider the service rate vary as service batches, the average response time will be delayed as the arrival rate increases, only when the batch satisfies the production of one cell. Sathiya, Ayyappan [13]compared the scheduling time under fixed service rate and variable service rate. When the service rate changes with the system state, the average response time is improved and the overall scheduling time is improved significantly. 
In this paper, due to the difference of the production process, we study the inter cell scheduling problem of the parts. Considering the factors of the batch size of the parts, the service rate and the arrival rate vary with the state of the system. Based on the queuing network theory, Cellular system is seen as a whole queuing network. The internal machine is seen as a service platform, and using the external arrival rate to calculate the parameters within the network. Considering the inter cell movements of parts, focusing on parts in different cells which need to wait in line, this study meet actual production needs.

\section{Model Formation}

\section{Problem Descriptions}

Assuming the virtual cellular manufacturing system of queuing network has $k$ service node, the node ${ }^{i(i=1,2, \ldots, k)}$ has ${ }^{c_{j}}$ service desk, service time of each service desk obey the exponential distribution, average service time of each service desk is ${ }^{1 /} \mu_{j}$, the parts reach the node at the rate of $v_{i}$, when the parts finished in the node $i(i=1,2, \ldots, k), r_{j, k}$ is the probability of leaving at the machine, arriving at the node $k$ to accept processing, all nodes can accept a certain number of parts for processing, when a part is completed, then transfer to another service node, which is independent with the service process that the part has received, and is independent with the service node where the other services are located. The queuing network system is a Jackson queuing network. If the queuing network system accepts parts out of the system and the parts can leave, it is an open Jackson queuing network system.

\section{Assumptions}

The queuing network can be simplified as an open-loop queuing network. The basic assumptions are as follows:

(1) Arrival of parts obey Poisson distribution, service time obey exponential distribution;

(2) The parts arrive at the machine is independent of each other;

(3) Each part contains a number of processes, at least in two different cells to complete, and there are order constraints between processes;

(4)The capacity of tackle between inter cell transportation is sufficient, that is the parts can be immediately transported to the destination cell at any time;

(5) Machine processing capacity overlap, the processing of parts can be completed on different machines, the parts have flexible processing path. For any one process, in each unit at most only one machine can be processed;

(6) Each machine is non-interrupt;

(7) Does not consider the parts to seize, machine failure, the default of parts.

Model parameters:

$i$ __ Part index, ${ }^{i=1,2, \cdots}$

j__ Machine index, ${ }^{j=1,2, \cdots, M}$

$h$ _Operation index, ${ }^{h=1,2, \cdots}$

$q_{i, j}$ _ processing time of part $i$

$\mu_{j} \_$Service rate

$\lambda_{j}$ __ Arrival rate

$b_{j}$ _Batch size

$r_{j, k} \_$Probability of processing

$S_{i, j}$ _ Start machining time

$C_{i, j}$ _Actual processing time

$W_{i, j}$ _Waiting time 
$c_{j}$-Number of cells

Model Variables:

$$
\begin{aligned}
\gamma_{\mathrm{i}, \mathrm{j}} & = \begin{cases}1, & \text { The part } i \text { is machined on machine } j \\
0, & \text { other }\end{cases} \\
a_{i, j, h, j, h^{\prime}} & =\left\{\begin{array}{ll}
1, & \text { Process } o_{i, j, h} \\
0, & \text { other }
\end{array} \text { takes precedence over process } o_{i, j, h^{\prime}}\right.
\end{aligned}
$$

\section{Queuing Network Model of Batch Service}

Virtual cellular manufacturing system of queuing network almost no feedback, that is, after t parts finishing processing, which go to the next node for processing directly. There is little rework to return to the previous node, unless there is a case of emergency. This article does not consider such special circumstances, so this queuing network system can be solved using the Jackson network system.

In the open queuing network system, the network path rules $\left\{r_{i j}, i(i=1,2, \ldots, k) ; j(j=1,2, \ldots . ., k)\right\}$ is:

$$
\sum_{i=0}^{k} r_{i j}=1,(j=1,2, \ldots . ., k)
$$

With the total arrival rate of $\lambda_{i}$ in the nodes $i$, the total arrival rate and the total output rate of the parts at each node in a stable system should be equal. $\lambda_{i}$ is equal to the sum of the arrival rates from other nodes and the external arrival rate of the system.

$$
\lambda_{i}=v_{i}+\sum_{j=1}^{k} r_{j i} \lambda_{j}(i=1,2, \ldots, k)
$$

According to the nature of Jackson's queuing network, the transition probability between nodes of the system form the vector matrix of the arrival rate of node, which can be used to calculate the total reach rate of each node? Assuming that $\mathrm{R}$ is the transfer probability matrix between the nodes in the Jackson network. $v_{i}$ is the vector of the arrival rate of each part from the outside of the network system to each node, then the vector of the actual arrival rate of each node is $\lambda=\left(\lambda_{1}, \lambda_{2}, \ldots, \lambda_{k}\right)^{\mathrm{T}}$, which meet $\lambda=\gamma(1-R)^{-1}$.

In the virtual cellular manufacturing system, the arrival probability of each node is calculated, since the part can be transferred to the service desk of another node after finishing processing. It depend on the processing capacity of the equipment and the actual completion time of the part. So the probability of part transferred can be calculated by the processing capacity of the equipment and the actual processing time of the part, that is $r_{i j}=C_{i j} / q_{i j}(i=1,2, \ldots, k ; j=1,2, \ldots, M) \quad$. So the total arrival rate of each node can be calculated, and then using the queuing network model formula to calculate other indicators.

The total machining flow time of the part is minimized, and the maximum load of the machine is minimized as the objective function:

$$
\begin{aligned}
f_{1}=\mathrm{T} & =\min \left(\sum_{i=1}^{N} \sum_{j=1}^{M}\left[\gamma_{i, j}\left(C_{i, j}+W_{i, j}\right)\right]\right) \\
f_{2} & =\min \left(\max _{1 \leq j \leq M} \sum_{i=1}^{N} \sum_{j=1}^{M} q_{i, j} \times \gamma_{\mathrm{i}, j} \times b_{j}\right)
\end{aligned}
$$


Constrain:

$$
\begin{aligned}
& S_{i, j}+\lambda_{i, j, h} \times b_{j} \times \gamma_{i, j, h} \times q_{i, j, h} \leq C_{i, h} \\
& C_{i, h} \leq S_{i,(h+1)} \\
& S_{i, h}+q_{i, j, h} \times b_{j} \leq S_{i^{\prime}, h^{\prime}}+M\left(1-a_{i, j, h . j^{\prime}, h^{\prime}}\right) \\
& C_{i, h} \leq S_{i, h+1}+M\left(1-a_{i, j^{\prime}, h^{\prime}, j,(h+1)}\right) \\
& \sum_{j=1}^{M} \gamma_{i, j}=1, \quad i=1,2, \cdots \\
& \sum_{i^{\prime}=1}^{n} \sum_{h^{\prime}=1}^{h_{i}^{\prime}} a_{i, j, h, j^{\prime}, h^{\prime}}=\gamma_{i, j, h} \\
& S_{i, h} \geq 0, \quad C_{i, h} \geq 0, \quad \lambda_{j}>0, \quad j=1,2, \cdots, N \\
& \mu_{j}=1 / \frac{\sum_{j=1}^{N} b_{j} q_{i, j}}{\sum_{j=1}^{N} c_{j}} \\
& \sum_{k=0}^{N} r_{j, k}=1, j=1, \cdots, N \\
& \mathrm{~W}_{j}=\frac{\lambda_{j}}{\mu_{j}\left(\mu_{j}-\lambda_{j}\right)}, \quad \forall j \\
& \mathrm{~W}_{s}=\frac{1}{\mu_{j}-\lambda_{j}}, \forall j
\end{aligned}
$$

In constraints, Equation (3) shows that the total machining flow time of the part is minimized in the network. Equation (4) shows minimize the maximum load in the network. Equation (5) and Equation (6) represent the sequence of each part in the network. Equations (7) and (8) show that the same machine in the network can only complete one process at the same time. Equation (9) shows that a process in the network can only be processed on one machine at the same time. Equations (10) show that the same machine in the network can be processed cyclically. Equation (11) is a nonnegative constraint. Equation (12) shows service rate constraint of the machine. Equation (13) shows the processing probability of the machine in the network. Equations (14) and (15) represent waiting time of the parts at any machines and time of staying.

\section{The Immune Optimization Algorithm}

The encoding is used here. Each process path scheme for parts can form an antibody with a length $\mathrm{p}$ (p represents the processing step). The issues raised in this paper involve allocating, scheduling, and processing batch sizes. Thus, each solution can be represented by the chromosome in three parts:

(1) Machine Code: This gene indicates the machine code in which the process is assigned to the machine for processing. 
(2) The code of the parts sequence: The chromosomes represent the feasible operation of the parts sequence.

(3) The code of batch size: that the batch size of each part.

As shown in Figure 1, information of the chromosome encoding can be expressed as $O_{12}$ processed on machine ${ }^{M_{33}}$, the batch of part I are 20 .

\begin{tabular}{|c|c|c|c|c|c|c|c|c|c|c|}
\hline sequence & $o_{1,1}$ & $o_{1,2}$ & $o_{2,1}$ & $o_{2,2}$ & $o_{2,3}$ & $o_{2,1}$ & $o_{1,1}$ & 1,2 & 2,2 & 2,3 \\
\hline machine & $M_{2,1}$ & $M_{3,1}$ & $\mathrm{M}_{2,2}$ & $M_{3,3}$ & $M_{1,1}$ & $M_{3,2}$ & $M_{4,1}$ & $\mathbf{M}_{1,2}$ & $M_{4,2}$ & M 5,1 \\
\hline code & 3 & 2 & 1 & 2 & 2 & 2 & 1 & 1 & 2 & 2 \\
\hline part & 1 & 2 & 3 & & & & & & & \\
\hline batch & 13 & 16 & 11 & & & & & & & \\
\hline
\end{tabular}

Fig.1 encoding

\section{Case Analysis}

\section{Case and Data}

In this paper, a group of machining workshops processing data in shipyard as an example to study the inter cell scheduling. A batch of orders have arrived, cluster decomposition of parts according to the processing, the parts need to go through five processes .This paper selects 16 parts, each part need to be processed across different cells.Basic information is shown in Table 1, Table 2, Table 3 and Table 4.

Tab.1 The workspace machine information

\begin{tabular}{|l|l|l|l|l|}
\hline Machine & Style & Number & Arrival rate & Service rate \\
\hline A & A1 & 1 & 0.6 & 10 \\
\hline B & B1,B2,B3 & $1,1,1$ & $0.3,0.7,0.6$ & $8,6,10$ \\
\hline C & C1,C2,C3 & $1,1,1$ & $0.8,0.2,0.5$ & $12,14,9$ \\
\hline D & D1,D2,D3 & 2,1 & $0.4,0.6,0.5$ & $16,13,14$ \\
\hline E & E1,E2 & 2 & $0.3,0.6$ & 10,11 \\
\hline
\end{tabular}

Tab.2 Job processing information

\begin{tabular}{|c|c|c|}
\hline Part & Machine/Processing time & Batch \\
\hline P1 & (C2-1.7)---(D1-2.7)---(E2-1.3)---(B1-2.1) & 6 \\
\hline P2 & (B2-1.9)---(E1-4.2) & 10 \\
\hline P3 & (B2-2.2)---(A1-3.3)---(C3-2) & 12 \\
\hline P4 & (C2-4.1)---(B1-5.6)---(D2-3) & 13 \\
\hline P5 & (C1-2.1)---(B3-4.3)---(D3-2.6) & 8 \\
\hline P6 & (C2-1.3)---(B1-2.1)---(D1-3.7)---(E1-4.5) & 9 \\
\hline P7 & (A1-6.8)---(C3-3.1) & 10 \\
\hline P8 & (D2-4.4)---(C1-1.9) & 11 \\
\hline P9 & (B3-2.7)---(E1-4.7) & 8 \\
\hline P10 & (B2-1.6)---(C3-1.3)---(E2-6.6) & 10 \\
\hline P11 & (D3-1.9)---(B2-2.3) & 9 \\
\hline P12 & (B1-3.4)---(C1-3.6)---(E2-1.1)---(D1-2.6) & 11 \\
\hline P13 & (D2-4.2)---(B2-7.3) & 7 \\
\hline P14 & (C3-4.5)---(D3-2.9)---(E1-4) & 6 \\
\hline P15 & (A1-1.9)---(D2-3.6) & 8 \\
\hline P16 & (C1-6.3)---(B1-1.9)---(D3-3.3) & 9 \\
\hline
\end{tabular}


Tab.3 Virtual cell information

\begin{tabular}{|l|l|l|l|l|}
\hline Scheme & Cell1 & Cell2 & Cell3 & Cell4 \\
\hline Machine & B3,E1 & $\begin{array}{l}\text { A1,B2,C3, } \\
\text { D2,D3 }\end{array}$ & B1,C1,E2 & C2,D1 \\
\hline Part & P2,P9 & $\begin{array}{l}\text { P3,P7,P10,P11 } \\
\text { P13,P14,P15 }\end{array}$ & P5,P8,P12,P16 & P1,P4,P6 \\
\hline
\end{tabular}

\section{Discussion}

The scheduling Gantt chart of the parts shown in Figure 2.

Tab.4 Job scheduling path and time of virtual cells

\begin{tabular}{|l|l|l|}
\hline Part & Path(time) & Cells \\
\hline P1 & C2(0-2.1)---D1(2.1-3.8)---E2(3.8-6.5)---B1(13-14.3) & Cell4, Cell3 \\
P2 & B2(1.6-3.5)---E1(3.5-7.7) & Cell2,Cell1 \\
P3 & B2(5.4-8.7)---A1(9.7-11.9)---C3(11.9-13.4) & Cell2 \\
P4 & C2(4.2-9.8)---B1(14.3-18.4)---D2(18.4-21.4) & Cell4, Cell3, Cell2 \\
P5 & C1(1.9-6.2)---B3(6.2-8.3)---D3(14.5-17.1) & Cell3,Cell1,Cell2 \\
P6 & C2(2.1-4.2)---B1(8.3-9.6)---D1(9.6-13.3)---E1(13.3-17.8) & Cell4,Cell3,Cell1 \\
P7 & A1(1-7.8)---C3(7.8-10.9) & Cell2 \\
P8 & D2(7.3-9.2)---C1(9.2-13.6) & Cell2,Cell3 \\
P9 & B3(0-2.7)---E1(7.7-12.4) & Cell1 \\
P10 & B2(0-1.6)---C3(1.6-2.9)---E2(6.5-13.1) & Cell2,Cel3 \\
P11 & D3(0-2.3)---B2(3.5-5.4) & Cell2 \\
P12 & B1(9.6-13)---C1(13.6-17.2)---E2(17.8-19.8)---D1(19.8-20.9) & Cell3, Cell4 \\
P13 & D2(0-7.3)---B2(8.7-12.9) & Cell2 \\
P14 & C3(2.9-7.4)---D3(11.6-14.5)---E1(17.8-21.8) & Cell2, Cell1 \\
P15 & A1(7.8-9.7)---D2(9.7-13.3) & Cell2 \\
P16 & C1(0-1.9)---B1(2-8.3)---D3(8.3-11.6) & Cell3, Cell2 \\
& & 9 \\
\hline
\end{tabular}

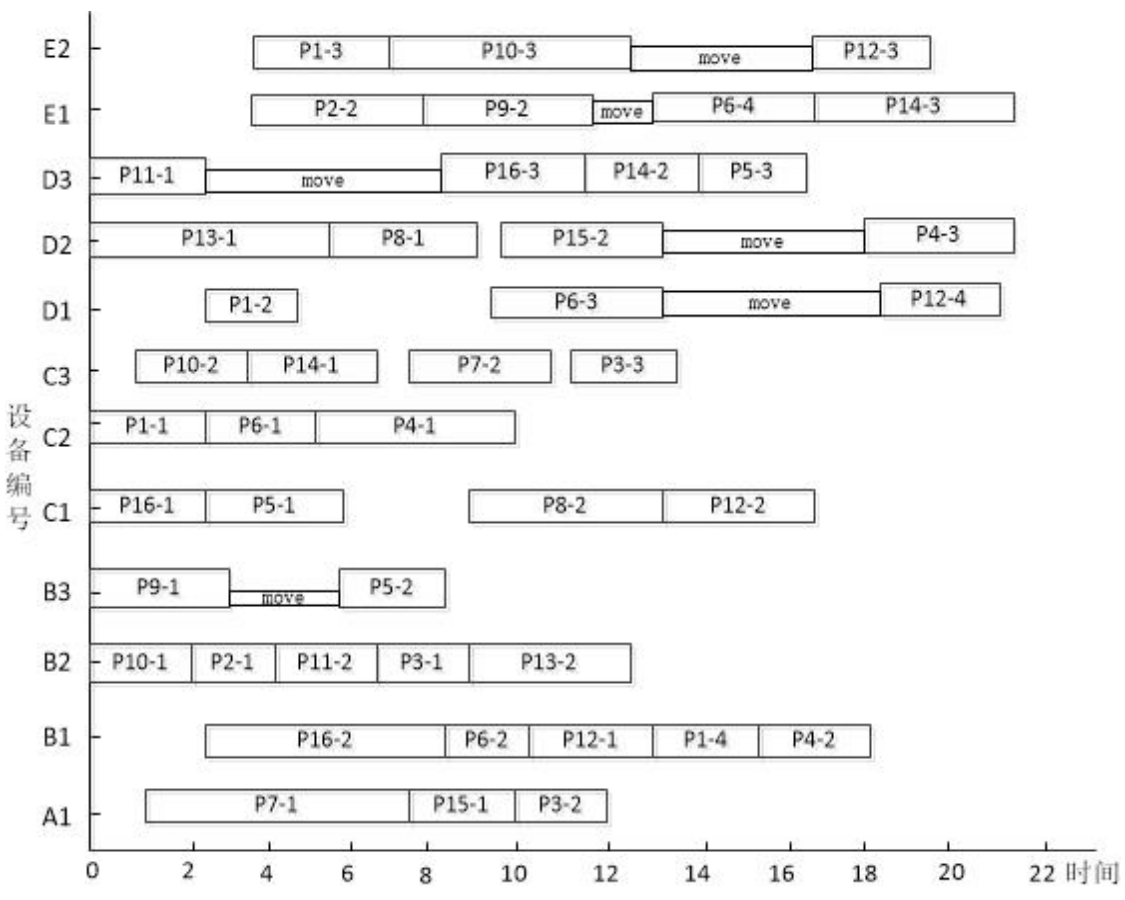

Fig.2 Scheduling chart

In the scheduling plan, the part 1 , the part 2 , the part 4 , the part 5 , the part 6 , the part 8 , the part 10 , the part 12 , the part 14 , the part 16 , due to the requirements of processing, they need to process in different cells, The part 7 , the part 9 , the part 11 , the part 13 , the part 15 does not need inter cell production. The maximum completion time of scheduling is 21.8 hours, and actual production time 
is 40 hours in enterprise. The scheduling time under queuing network is lower than that of the original production time, and the research has a certain significance.

\section{Conclusion}

The problem of cell scheduling is a very important scheduling problem in modern manufacturing management. In order to minimize the maximum completion time and minimize the maximum load of the machine, this paper studies the problem of inter cell scheduling, which is based on the queuing network method and the immune optimization algorithm. The service rate and the arrival rate of the queuing network are combined with the batches of parts. The nonlinear dynamic mathematical programming model is established to solve the problem of inter cell scheduling. The proposed algorithm is used to solve the case, and the scheduling is carried out by using MATLAB. The experimental results verify that the proposed algorithm is feasible and effective. This paper does not consider the bottleneck of the batch. The problem of inter cell scheduling has yet to be studied in depth. In the future, the paper construct model by combining with queuing network and batch.

\section{Acknowledgement}

This paper is sponsored by National Natural Science Foundation of China (GN: 71271105) and Humanities and Social Sciences Planning Fund of Ministry of Education (GN: 12YJA630036).

\section{References}

[1] Darla S P, Naiju C D, Sagar P V, et al. Optimization of inter cellular movement of parts in cellular manufacturing system using genetic algorithm[J]. Research Journal of Applied Sciences Engineering \& Technology, 2014, 7(1):165-168.

[2] Taha Z, Rostam S. A hybrid fuzzy AHP-PROMETHEE decision support system for machine tool selection in flexible manufacturing cell [J]. Journal of Intelligent Manufacturing, 2011, 23(6):2137-2149.

[3] Zeng C, Tang J, Yan C. Job-shop cell-scheduling problem with inter-cell moves and automated guided vehicles [J]. Journal of Intelligent Manufacturing, 2014, 26(5):1-15.

[4] Mak K L, Ma J, Su W. A constraint programming approach for production scheduling of multi-period virtual cellular manufacturing systems[C]// Sixth International Conference on Natural Computation. IEEE, 2010:4440 - 4444.

[5] Tang J, Yan C, Wang X, et al. Using Lagrangian Relaxation Decomposition With Heuristic to Integrate the Decisions of Cell Formation and Parts Scheduling Considering Intercell Moves[J]. IEEE Transactions on Automation Science \& Engineering, 2014, 11(4):1110-1121.

[6] R. Tavakkoli-Moghaddam, N. Javadian, A. Khorrami, Y. Gholipour-Kanani. Design of a scatter search method for a novel multi-criteria group scheduling problem in a cellular manufacturing system [J].Expert Systems with Applications. 2010(37): 2661-2669.

[7] Hasenbein J J. Necessary conditions for global stability of multiclass queueing networks [J]. Operations Research Letters, 1997, 21(2):87-94.

[8] Harrison J M, Nguyen V. Brownian models of multiclass queueing networks: Current status and open problems [J]. Queueing Systems, 1993, 13(1-3):5-40.

[9] Dai J G, Li C. Stabilizing Batch-Processing Networks [J]. Operations Research, 2003, 51(1):123-136.

[10] Wu Lihui, Zhang Jie. Modeling Method for Performance Analysis of Material Handling 
System in Wafer Fabrication [J]. Computer Integrated Manufacturing System, 2013, 19(8):2043-2049.

[11] Liu Li. Probabilistic Scheduling Algorithm for Cloud Session Based on Stochastic Service Model [J]. Journal of Qiqihar University (Natural Science Edition), 2015(2):65-70.

[12] Laxmi P V, Seleshi D. Discrete-Time State Dependent Bulk Service Queue with Multiple Vacations and Changeover Times [J]. Isrn Operations Research, 2014, 2014(1-2):1-12.

[13] Sathiya K, Ayyappan G. A NON-MARKOVIAN BATCH ARRIVAL QUEUE WITH SERVICE INTERRUPTION \& EXTENDED SERVER VACATION [J].International Journal of Mathematical Modelling \& Computations, 2015, 5(1). 